\title{
Extensive tabulations of Stark broadened hydrogen line profiles ${ }^{\star}$
}

\author{
C. Stehlé ${ }^{1, \star \star}$ and R. Hutcheon ${ }^{1, \star \star \star}$ \\ UMR 8629 du CNRS, DARC, Observatoire de Paris, 92195 Meudon Cedex, France
}

Received March 08; accepted September 16, 1999

\begin{abstract}
New tables of Stark broadened hydrogen Lyman, Balmer and Paschen lines are presented for a wide range of plasma conditions, allowing the complete spectrum of these series to be modelled up to the Inglis Teller limit. The formalism, based on the Model Microfield Method (MMM) for both the electronic and ionic broadenings, gives an accurate description of the line profile from the centre to the wings.
\end{abstract}

Key words: atomic data — line profiles — plasmas

\section{Introduction}

Hydrogen lines are of major interest in astrophysical problems. They are used, for example, to determine fundamental stellar parameters like effective temperature and surface gravity or to derive the electron density in solar flares. They also play a role in the macroscopic composition of stellar envelopes through the opacity and radiative diffusion processes, and also in the structure of stellar atmospheres.

These purposes require reliable tabulations extending from the line centres to the line wings. The first tables of Underhill \& Waddel (1959) were followed by those of Vidal et al. (1973), which neglected the effects of the ion motions in the ionic broadening ("static ion approximation") and used a simplified description of electronic broadening by neglecting the time ordering effects. These assumptions are known to underestimate the line centre broadening, especially at low densities. Nethertheless

Send offprint requests to: C. Stehlé

* The tables are only available in electronic form at the CDS via anonymous ftp to cdsarc.u-strasbg.fr (130.79.128.5) or via http://cdsweb.u-strasbg.fr/Abstract.html

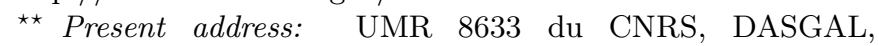
Observatoire de Paris, 92195 Meudon Cedex, France.

$\star \star \star$ Present address: DCTA, S+T Division, Flagstaff Road, Colchester, Essex CO2 7SS, UK. these tabulations give a correct description of the line wings and are extensively used for the spectral synthesis of stellar atmospheres. In this context, they allow satisfactory reproduction of the variation of the stellar flux with the wavelength. These tabulations have been recently extended by Lemke (1997) to Paschen and Brackett lines. They extend possibilities of the diagnostics of stellar atmospheres towards the infrared.

The reason why the VCS tables were successful for stellar atmospheres lies in the great importance of radiative transfer in the line centre, which smears out most of the line structure. This situation is different in low density media like solar flares where using these tables can overestimate the density. Moreover, developments in high resolution spectroscopy, may require also more accurate astrophysical modelling and therefore improved input physics. One of us tried to work in this direction by publishing tables of Lyman, Balmer and Paschen series for the plasma conditions of stellar envelopes (Stehlé 1994a; 1996a); these tables have also been parametrized for the two first series (Clausset et al. 1994). Unfortunately, these tables were restricted to a limited range of density and temperatures, and were not suitable for stellar atmosphere applications. This is why we present here new extended calculations for a density range between $10^{10}$ and $10^{19} \mathrm{~cm}^{-3}$ and temperatures between 2500 and $1.310^{6} \mathrm{~K}$. The extremes of these ranges of temperatures and densities are calculated essentially to allow more accurate interpolation in the tables.

\section{Description of the method}

The broadening of spectral lines by a plasma is due to the interactions between the radiating element and the free ions and electrons of the plasma. These two contributions can be described in terms of interaction potentials with the corresponding electronic and ionic plasma microfields. These microfields are stochastic processes, whose static properties are described by distribution functions $P(E)$. In the Model Microfield Method Frisch \& Brissaud (1971), 
the dynamics of the microfield fluctuations are treated by a statistical process model where the microfield (electronic or ionic) is assumed to be constant during a given time interval. The microfield then jumps instantaneously to another constant value for the next time interval. The jumping times follow Poisson statistics, with a field dependent frequency $\nu(E)$. The calculation requires the knowledge of the electronic and ionic field distribution functions, for which Hooper's distribution functions (Hooper 1966; Hooper 1968) were used. The other input parameter required is the jumping frequency $\nu(E)$, which is chosen so as to reproduce the field autocorrelation function correctly Brissaud \& Frisch (1971); Stehlé (1994b).

This model is the only universal model, currently available, which can reproduce correctly both the line widths and the intensity profile in the line wings. However, in the line centre, it might be interesting to compare the MMM linewidths with the results of computer simulations Gigosos \& Cardenoso (1996); Cardenoso \& Gigosos (1997), which do not include the Doppler broadening. Such simulations can reach accurate results for the line width. On the other hand, they suffer from two limitations, neither of which affect MMM modelling. First, perturbing charges (electrons and ions) are assumed to move along straight lines, which is incorrect at high densities. Secondly, the numerical procedure converges with difficulty at low densities and in the line wings, where the contributions of rare close interactions become important.

The present calculation, summarized below, follows the procedure already described by Stehlé (1994a, $1994 b)$. Let the initial and final states of the optical transition be denoted by $i, i^{\prime} \ldots$ and $f, f^{\prime} \ldots$, (with energies $\left.E_{i}, E_{i}^{\prime}, E_{f}, E_{f}^{\prime}\right)$. The line profile, normalized to unit area, $I(\omega)$, is defined in the Liouville space spanned by the states $\mid$ if $>>$ by

$I(\omega)=\frac{1}{\pi} \sum_{i f, i^{\prime} f^{\prime}} \boldsymbol{d}_{i f} \boldsymbol{d}_{i^{\prime} f^{\prime}}<U\left(\omega, \boldsymbol{F}_{i}, \boldsymbol{F}_{\mathrm{e}}\right)>_{e i ; i f, i^{\prime} f^{\prime}}$

where $\langle U(\omega)\rangle_{e i}$ is the Fourier transform of the hydrogen evolution operator, averaged over the realizations of the stochastic dynamic electronic and ionic microfields $\boldsymbol{F}_{i}, \boldsymbol{F}_{\mathrm{e}}$, and $\boldsymbol{d}$ is the dipole operator of the hydrogen bound electron (normalized to $\sum_{i f} d_{i f}^{2}$ ).

We shall use the "no quenching approximation", which means that the interactions between the plasma and the radiating element mix together only those eigenstates belonging to the same principal quantum number. Neither do we include the fine structure effects. Thus $E_{f}-E_{i}=$ $E_{f^{\prime}}-E_{i^{\prime}}=\hbar \omega_{0}$.

The typical fluctuation times of the ionic and electronic microfields, respectively given by the inverse of the electronic and ionic plasma frequencies $\omega_{\mathrm{pe}}, \omega_{\mathrm{pi}}$, are very different. Thus, during an interval of constancy of the ionic microfield $\boldsymbol{F}_{i}$, the electronic microfield presents large time fluctuations. This justifies averaging the evolution operator over these electronic fluctuations for a given ionic microfield value. The procedure has been applied to the Model Microfield Method by Brissaud \& Frisch (1971) and discussed by Frerichs (1989). It requires first calculating, for the static ionic microfield $\boldsymbol{F}_{i}$, the evolution operator $<U\left(\omega, \boldsymbol{F}_{i}\right)>_{\mathrm{e}}$, averaged over electrons, and then performing the dynamical MMM average over the ions.

In practice, we express $\left\langle U\left(\omega, \boldsymbol{F}_{i}\right)\right\rangle_{\mathrm{e}}$ in terms of the frequency dependent relaxation operator $\gamma_{\mathrm{e}}\left(\omega, \boldsymbol{F}_{i}\right)$ (Greene 1982) defined by

$<U\left(\omega, \boldsymbol{F}_{i}\right)>_{\mathrm{e}}=i\left[\Delta \omega I-\boldsymbol{d} \boldsymbol{F}_{i}+i \gamma_{\mathrm{e}}\left(\omega, \boldsymbol{F}_{i}\right)\right]^{-1}$

where $\Delta \omega$ is the detuning from the line center $\omega_{0}$.

It is thus equivalent whether we calculate $<U\left(\omega, \boldsymbol{F}_{i}\right)>_{\mathrm{e}}$ or $\gamma_{\mathrm{e}}\left(\omega, \boldsymbol{F}_{i}\right)$. We shall suppose that $\gamma_{\mathrm{e}}\left(\omega, \boldsymbol{F}_{i}\right)$ is independent of the ionic microfield value, or $\gamma_{\mathrm{e}}\left(\omega, \boldsymbol{F}_{i}\right)=\gamma_{\mathrm{e}}(\omega)$. This approximation is justified because the linear ionic splitting is almost negligible compared to the electronic plasma frequency.

To obtain $\gamma_{\mathrm{e}}(\omega)$, it is thus sufficient to calculate $<U\left(\omega, \boldsymbol{F}_{i}\right)>_{\mathrm{e}}$ for $F_{i}=0$. This electronic average is performed by the Model Microfield Method, using the appropriate electronic frequency jump $\nu_{\mathrm{e}}\left(F_{\mathrm{e}}\right)$ and the MMM equation for $\langle U\rangle_{\mathrm{e}}$,

$$
\begin{aligned}
<U(\omega)>_{\mathrm{MMM}, \mathrm{e}} & =\left\{U\left(\omega+i \nu_{\mathrm{e}}\right\}+\left\{\nu_{\mathrm{e}} U\left(\omega+i \nu_{\mathrm{e}}\right)\right)\right\} \\
& \times\left\{\nu_{\mathrm{e}} I-\nu_{\mathrm{e}}^{2} U\left(\omega+i \nu_{\mathrm{e}}\right)\right\}^{-1} \\
& \times\left\{\nu_{\mathrm{e}} U\left(\omega+i \nu_{\mathrm{e}}\right)\right\}
\end{aligned}
$$

where $U\left(\omega+i \nu_{\mathrm{e}}\left(F_{\mathrm{e}}\right)\right)$ is defined by

$U\left(\omega+i \nu_{\mathrm{e}}\left(F_{e}\right)\right)=i\left(\Delta \omega I-\boldsymbol{d} . \boldsymbol{F}_{\mathrm{e}}+i \nu_{\mathrm{e}}\left(F_{\mathrm{e}}\right) I\right)^{-1}$.

The notation $\{.$.$\} denotes the static average over the elec-$ tronic plasma microfield $P_{\mathrm{e}}\left(F_{\mathrm{e}}\right)$ (i.e. $\left.\int_{0}^{\infty} P_{\mathrm{e}}\left(F_{\mathrm{e}}\right) \ldots \mathrm{d} F_{\mathrm{e}}\right)$ and $\nu_{\mathrm{e}}\left(F_{\mathrm{e}}\right)$ is defined by

$\nu_{\mathrm{e}}\left(F_{\mathrm{e}}\right)=\frac{\omega_{\mathrm{pe}}}{x+1}\left[(40 x)^{1 / 5}+x(x+1)+\frac{3}{2}\left(\frac{\pi}{2}\right)^{1 / 2}\right]$

with

$x=x\left(F_{\mathrm{e}}\right)=\int_{0}^{F_{\mathrm{e}}} E^{2} P_{\mathrm{e}}(E) \mathrm{d} E$.

From $<U(\omega)>_{\text {MMM,e }}$ the electronic damping rate $\gamma_{\mathrm{e}}(\omega)$ is extracted, using the relation 2 , with $F_{i}=0$. The dynamic average over all the realizations of the ionic microfields is then performed in the frame of the Model Microfield Method. The method and equations are similar to the previous ones, if one uses the appropriate ionic frequency jump $\nu_{i}\left(F_{i}\right)$, (Eq. (17) of Stehlé (1994b), with correction of the erroneous factor $(160 x)^{1 / 5}$ which should be replaced by $\left.(40 x)^{1 / 5}\right)$, the ionic field distribution function $P_{i}$ and the following expression of $U\left(\omega+i \nu_{i}\left(F_{i}\right)\right)$ for the fixed value of the ionic field $\boldsymbol{F}_{i}$,

$U\left(\omega+i \nu_{i}\left(F_{i}\right)\right)=i\left[\Delta \omega I-\boldsymbol{d} . \boldsymbol{F}_{i}+i\left(\gamma_{\mathrm{e}}(\omega)+\nu_{i}\left(F_{i}\right) I\right)\right]^{-1} \cdot(7)$

One then obtains the plasma averaged evolution operator $<U(\omega)>_{\mathrm{MMM} \text {,ei }}$ which includes the contributions of both the ions and the electrons. The final Stark profile $I(\omega)$ is 
obtained after weighting of the averaged plasma evolution operator $\langle U(\omega)>$ using Eq. (1).

In general $\gamma_{\mathrm{e}}(\omega)$ is not diagonal in if and $i^{\prime} f^{\prime}$. This leads in practice to the numerical inversion of a non diagonal matrix, which is difficult to perform when the final state has a large principal quantum number. In this case, in order to make the calculation more tractable, we perform the "isotropic" calculation, in which $\gamma_{\mathrm{e}}(\omega)$ is approximated by its scalar mean defined as

$\bar{\gamma}_{\mathrm{e}}(\omega)=\sum_{i f, i^{\prime} f^{\prime}} \boldsymbol{d}_{i f} \boldsymbol{d}_{i^{\prime} f^{\prime}} \gamma_{\mathrm{e} ; i f, i^{\prime} f^{\prime}}(\omega) / \sum_{i f} \boldsymbol{d}_{i f} \boldsymbol{d}_{i f}$

This approximation gives exactly the same line wings intensity as the "standard" calculation. At low densities, it has been proved (Stehlé 1996a; Cassini 1997), that the centres of hydrogen lines are lorentzian, with an halfwidth equal to $\bar{\gamma}(\omega)$. Thus this isotropic approximation has a (small) effect only in the line centres and at large densities.

Even in this approximation, the calculation may be too time consuming and one uses the "isotropic, with no lower state interaction" approximation, but in a different way from that used by Stehlé (1994a). In both the present calculation and in Stehlé (1994a), this approximation means essentially that the lower state broadening is negligible compared with that of the upper state. In Stehlé (1994a) expression (1) was evaluated for all the dipole matrix elements $d_{i f} d_{i^{\prime} f^{\prime}}$ of the optical transitions. Our method, in the present paper, differs in that we impose a more severe approximation, by assuming that for large $n^{\prime}$, the line shape (without Doppler broadening) does not depend at all on the lower level $n$ of the transition. This approximation also means that for the case where the Doppler broadening is negligible (i.e. at large densities), the Lyman $n^{\prime}$, Balmer $n^{\prime}$ and Paschen $n^{\prime}$ transitions have the same profile. Doppler broadening must be fully taken into account, as this obviously depends on the line. The error introduced by this approximation has been discussed by Vidal et al. (1971) and Lemke (1997) in the static ion approximation. We illustrated the effects of the approximation, in the context of the MMM, for the Balmer 6, 7 and 8 transitions in Fig. 1.

In practice, we have carried out the calculation of the Lyman 2, 3, 4, 5 and Balmer 3, 4, 5 with the "anisotropic" approximation, and that for the Lyman 6 and higher, for Balmer 6 to 7 and for Paschen 4 to 8 with the "isotropic" approximation. The higher Balmer and Paschen lines are approximated by "Lyman" Stark profiles convolved (if necessary) by the appropriate Doppler broadening.

For any given density, there is an effective series limit beyong which individual lines are no longer resolved. This limit falls with density and can be estimated qualitatively by the Inglis-Teller formula (Inglis \& Teller 1939; Vidal 1966), as

$\log _{10}\left(n_{\mathrm{IT}}\right)=3.10-0.13 \log _{10}\left(2 N_{\mathrm{e}} / \mathrm{cm}^{-3}\right)$.

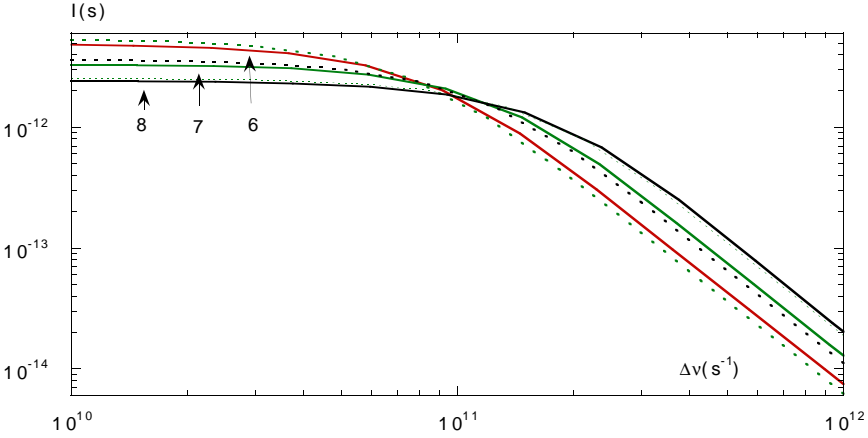

Fig. 1. The line shapes of Ly6, Ly7, Ly8 (full lines) and H6, H7, H8 (dashed lines), without Doppler broadening, in the "isotropic" approximation for $N_{\mathrm{e}}=10^{14} \mathrm{~cm}^{-3}$ and $T=$ 10000 K. Detunings are given in frequency units

The present calculations are carried out for the Lyman and Balmer lines up to the Inglis-Teller limit for 20 electronic densities $N_{\mathrm{e}}$ (given by $\log _{10}\left(N_{\mathrm{e}}\left(\mathrm{cm}^{-3}\right)\right)=10,10.5,11, \ldots$ $18,18.5,19,19.5$ ), and 10 temperatures $T$ in $\mathrm{K}$ (given by $\left.\log _{10}(T(\mathrm{~K}))=3.4,3.7,4.0 \ldots 5.5,5.8,6.1\right)$.

Although the MMM can perform line shape calculation for correlated plasmas, the present tabulation is limited to density-temperature range where the mean interelectronic distance $r_{0}$ is smaller that the electronic Debye length $\lambda_{\mathrm{d}}$. This allows the use of Hooper's high frequency and low frequency field distribution functions Hooper (1966); Hooper (1968), which are necessary to calculate respectively the electronic and ionic contributions to the line. Thus tabulations are limited to the density-temperature range, where

$a=\frac{r_{0}}{\lambda_{\mathrm{d}}}=0.0898 \frac{\left(N_{\mathrm{e}} / \mathrm{cm}^{-3}\right)^{1 / 6}}{(T / \mathrm{K})^{1 / 2}}<1$.

\section{Use of the tables}

Whatever quantity is chosen as the detuning parameter, the intensity profile must be area normalized. The change from wavelength $\lambda$ to angular frequency $\omega$ is then given by

$I(\lambda)=I(\omega)|\mathrm{d} \omega / \mathrm{d} \lambda|$ with $\omega=2 \pi c / \lambda$.

The physical choice of parameter for the the line shape calculation is derived energy detunings (i.e. frequency, angular frequency, wavenumbers, energy...). For Stark broadening, it may also be convenient to normalize this detuning by the normal Holtsmark field $F_{0}$ defined by

$$
\begin{aligned}
F_{0} & =e / r_{0}^{2} \\
F_{0} / \mathrm{esu} & =1.2510^{-9}\left(N_{\mathrm{e}} / \mathrm{cm}^{-3}\right)^{2 / 3}
\end{aligned}
$$

where $r_{0}$, closely equal to the mean interelectronic distance is defined by

$(4 / 15)(2 \pi)^{3 / 2} r_{0}^{3} N_{\mathrm{e}}=1$.

An appropriate choice for the detuning parameters for Stark broadening calculations would be $\Delta \Omega=\Delta \omega / F_{0}$ 
(units: $\mathrm{rad} \mathrm{s}^{-1} \mathrm{esu}^{-1}$ ). Despite this, parameters based on wavelength are often preferred for practical applications. Previous tabulations (Vidal et al. 1973; Stehlé 1994a; Stehlé 1996a; Lemke 1997) used reduced wavelength detunings defined as $\alpha$ units $\left(\AA \mathrm{esu}^{-1}\right)$ where

$\Delta \alpha /\left(\AA_{\mathrm{esu}^{-1}}\right)=\frac{(\Delta \lambda / \AA)}{\left(F_{0} / \mathrm{esu}\right)}$.

As functions of energy or angular frequency detunings, the line shapes are symmetrical around the line center, i.e. $I(-\Delta \omega)=I(\Delta \omega)$. The transformation of this relation in wavelength detunings, should allow for the albeit small asymmetry introduced by this choice of detunings, that is we use

$\Delta \lambda=-2 \pi c \frac{\Delta \omega}{\omega_{0}\left(\omega_{0}+\Delta \omega\right)}$

where $\omega_{0}$ and $\lambda_{0}$ are respectively the angular frequency and the wavelength of the unperturbed transition and $\omega_{0}=2 \pi c / \lambda_{0}$. This "trivial" asymmetry must be distinguished from the "intrinsic" asymetry, which is not included in this calculation. Hydrogen line "intrinsic" asymmetry is negligible for modelling stellar atmospheres. It is due to different competitive mechanisms like for example fine structure effects (which affects the line centers at low densities, Stehlé \& Feautrier 1985), and quantum and short range effects (see for example, Demura \& Sholin 1975; Feautrier et al. 1976; Stehlé 1986; Demura et al. 1990; Döhrn et al. 1996; Günter \& Könies 1997) which mainly affect the line wings. These asymmetry sources are not related to those observed in moving media.

In order to be consistent with earlier tabulations, we performed the tabulation using reduced wavelength $\Delta \alpha$ detunings. For each transition, the line shapes tabulations corresponding to different electronic densities are reported in separate files. They are given for positive (wavelength) detunings, before and after Doppler convolution. The line wing parameter $K_{\alpha}$ is also given. It allows the line shape to be extrapolated towards large detunings, the relation

$I(\Delta \omega)=\frac{K_{\omega}}{(\Delta \omega)^{5 / 2}}$

or in reduced wavelengths,

$$
\begin{aligned}
& I(\Delta \alpha)=\frac{K_{\alpha}}{(\Delta \alpha)^{5 / 2}} \times\left(\frac{\lambda_{0} / F_{0}+\Delta \alpha}{\lambda_{0} / F_{0}}\right)^{1 / 2} \\
& K_{\alpha}=K_{\omega} \times\left(\frac{\lambda_{0}}{2 \pi c}\right)^{3} .
\end{aligned}
$$

Concerning the transitions from a highly excited level $n^{\prime}$, in "no lower state interaction", the intensity profiles of the Balmer, Paschen... and Lyman lines, in angular frequency detunings, are (before Doppler convolution) exactly the same. Thus the factor $K_{\omega}$ is invariant.

\section{Discussion}

We shall not present here detailed comparisons with availaible experimental results, since these may be found in Stehlé (1994a) and (1996a) for individual lines, and in Stehlé \& Jacquemot (1993), for the comparison between the Balmer series emissivity obtained with MMM profiles and the experimental results. The examples presented in these papers show that the line shape computation is accurate to better than $10 \%$. It differs from one line to another and from the line center to the line wings. The MMM approach slightly underestimates the ion dynamics effects in the line centres at low densities. Moreover, the description of short range interactions must be improved in the far wings or at large densities.

Spontaneous emission and fine structure effects are not included in the calculation. For a given electronic density and temperature, these effects are more important for the lowest lines of the series. The broadening effect of spontaneous decay can to a good approximation be introduced through a convolution with a Lorentz profile. Fine structure effects can also be qualitatively introduced through a convolution with the corresponding Dirac structure spectrum.

Broadening due to the interaction with neutral hydrogen atoms (or other atoms), leading to Van der Waals and resonance broadening are not included here, but could be by convolving the corresponding profile with the Stark profile.

To illustrate the new possibilities offered by the present extended tabulations, we calculated the emissivity of two different plasmas near the Balmer series limit, assuming that both are in Local Thermodynamical Equilibrium, and using the method described in Stehle \& Jacquemot (1993). These two cases are first, an hydrogen plasma with an electronic density equal to $10^{13} \mathrm{~cm}^{-3}$ and a temperature of $10000 \mathrm{~K}$ (solar chromospheric plasma) and secondly 4.5 $10^{14} \mathrm{~cm}^{-3}$ and $44000 \mathrm{~K}$ (Figs. 2, 3).

The first case (Fig. 2) shows a large number of resolved lines. Each of them could provide information about the plasma conditions. The diagnostics will be more tightly constrained by using a part of the spectrum including several lines.

The same conclusions can be drawn in the second example (Tokamak case). The conditions have been chosen in order to reproduce the experimental line widths of the $2-8,2-9,2-10$ and $2-11$ transitions (i.e. H8, H9, H10 and H11) given by Welch et al. (1995). After deconvoltion from experimental broadening, these authors obtain full half widths of $4.7,5.9,7.5$ and $9.6 \AA$ respectively. We were able to model $4.6,5.8,7.5$ and $9.4 \AA$ for parameters of $4.510^{14} \mathrm{~cm}^{-3}$ and $44000 \mathrm{~K}$. The spectrum (Fig. 3) corresponds qualitatively with the experimental ones. The H11 transition is partly merged with the H12 ones, which makes it more difficult to determine the half width. Our determination is only indicative, and ignores the fact that 


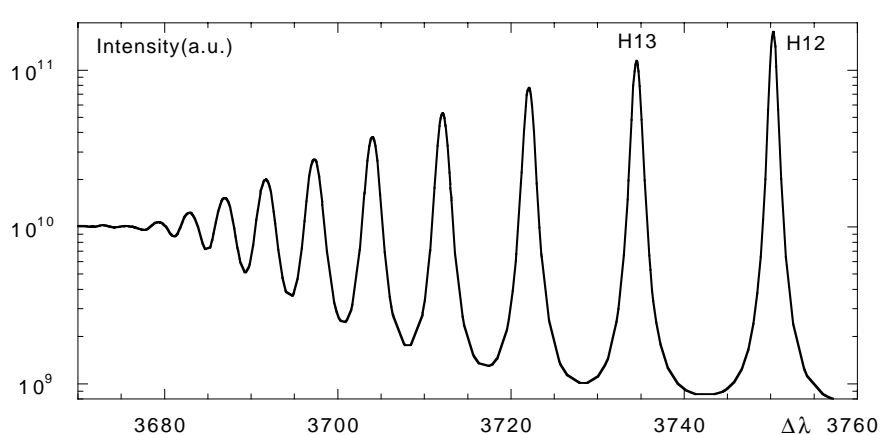

Fig. 2. LTE emissivity of an hydrogen plasma at $N_{\mathrm{e}}=$ $10^{13} \mathrm{~cm}^{-3}$ and $T=10000 \mathrm{~K}$

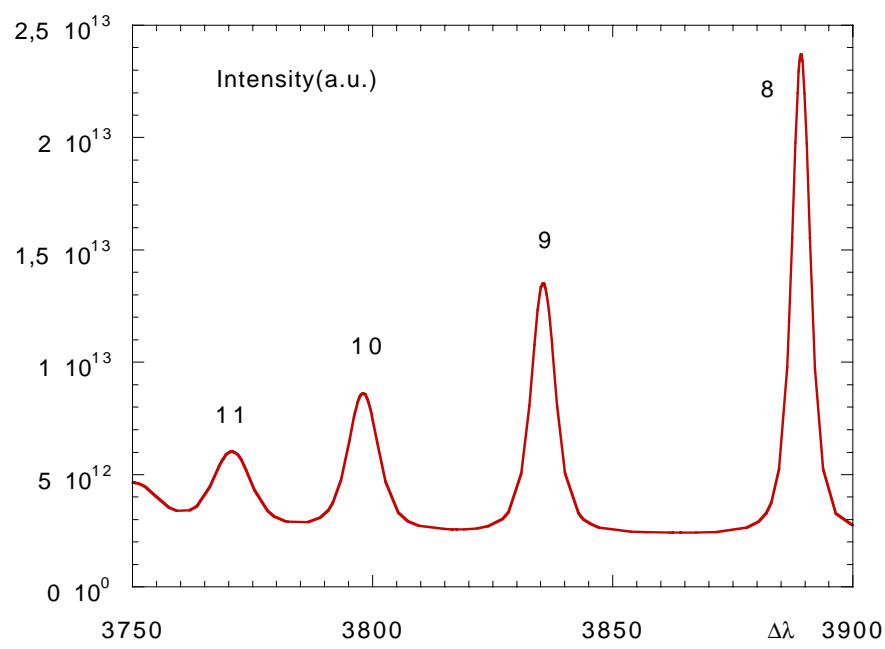

Fig. 3. LTE emissivity of an hydrogen plasma at $N_{\mathrm{e}}=4.5$ $10^{14} \mathrm{~cm}^{-3}$ and $T=44000 \mathrm{~K}$

the experimental plasma is a plasma of deuterium instead of hydrogen. Thus Doppler broadening is smaller in the experiment (FWHM of the order of $0.3 \AA$ for deuterium at $3900 \AA$ for $0.4 \AA$ for hydrogen). Nethertheless it is interesting to note that our calculation gives a smaller electronic density than those obtained by these authors. This point has been underlined by Welch et al. (1997).

Acknowledgements. R.H. acknowledges the support of the European Network Human Capital and Mobility ERBCHRXTCT 930377. Most of the computations have been performed on the Cray C94 at IDRIS, France, whith the help of J. Chergui for the implementation of the codes. The authors are also grateful to the referee for his constructive comments.

\section{References}

Brissaud A., Frisch U., 1971, J. Quant. Spectrosc. Rad. Transfer 11, 1767

Cardenoso V., Gigosos M.A., 1997, J. Phys. B: At. Mol. Phys. 30,3361

Casini R., 1997, J. Math. Phys. 38, 3435, erratum J. Math. Phys. 1997, 38, 6040

Clausset F., Stehlé C., Artru M.C., 1994, A\&A 287, 666

Demura A.V., Sholin G.V., 1975, J. Quant. Spectrosc. Rad. Transfer 15, 881

Demura A.V., Pleshakov V.V., Sholin G.V., 1990 (preprint IAE-5346/6)

Döhrn A., Nowack P., Könies A., Günter S., Helbig V., 1996, Phys. Rev. E 53, 6389

Feautrier N., Tran Minh N., Van Regemorter H., 1976, J. Phys. B: At. Molec. Phys. 9, 1871

Frerichs M.R., 1989, Z. Phys. D, Atoms, Molecules Clusters 11,315

Frisch U., Brissaud A., 1971, J. Quant. Spectrosc. Rad. Transfer 11, 1753

Gigosos M.A., Cardenoso V., 1996, J. Phys. B: At. Mol. Phys. 29,4795

Greene R.L., 1982, J. Quant. Spectrosc. Rad. Transfer 27, 185

Günter S., Könies S., 1997, Phys. Rev. E 55, 907

Hooper C.F., 1966, Phys. Rev. 149, 77

Hooper C.F., 1968, Phys. Rev. 165, 215

Inglis D.R., Teller E., 1939, ApJ 90, 439

Lemke M., 1997, A\&A 122, 285

Stehlé C., Feautrier N., 1985, J. Phys. B: At. Molec. Phys. 18, 1297

Stehlé C., 1986, Phys. Rev. A 34, 4153

Stehlé C., Jacquemot S., 1993, A\&A 271, 348

Stehlé C., 1994a, A\&AS 104, 509

Stehlé C., 1994b, A\&A 292, 699

Stehlé C., 1996a, Phys. Scr. 1996, T65, 183

Stehlé C., 1996b, A\&A 305, 677

Underhill A.B, Waddel J.H., 1959, NBS Circ. 603

Vidal C.R., 1966, J. Quant. Spectrosc. Rad. Transfer 6, 461

Vidal C.R., Cooper J., Smith E.W., 1971, NBS Monograph 120

Vidal C.R., Cooper J., Smith E.W., 1973, ApJS 25, 37

Welch B.L., Griem H.R., Terry J., Kurz C., LaBombard B., Lipschultz B., Marmar E., McCracken G., 1995, Phys. Plasmas 2, 4246

Welch B.L., Griem H.R., Weaver J.U., et al., 1997, in "Spectral Line Shapes", Vol. 9, Zoppi M., Ulivi L. (eds.), AIP Conf. Proc. 386, New York, p. 113 